\title{
Bird migration
}

\author{
Franz Bairlein ${ }^{1}$ Wolfgang Wiltschko ${ }^{2}$
}

Published online: 14 June 2017

(C) Springer-Verlag Berlin Heidelberg 2017

When Friedrich Barth, the editor-in-chief of the Journal of Comparative Physiology A, invited us to become the guest editors of a Special Issue entitled "Bird Migration", we immediately accepted his offer. The migration of birds is a really fascinating subject. No other group of terrestrial animals is as mobile as the birds are. Their ability to fly enables them to move around in large home ranges, and this might have predisposed them to undertake their extended migratory journeys. The annual migration of globally billions of birds is one of the most spectacular phenomena observed in nature. Migrations between breeding and non-breeding sites range from a few hundred kilometres in short-distance migrants such as goldfinches and siskins to impressive tens of thousands of kilometres each year in long-distance migrants. Examples of such long-distance migrants are the bar-tailed godwit, which travels from Alaska to New Zealand, the tiny northern wheatear going 15,000 km from Alaska across Asia into eastern Africa and the Arctic tern, which travels from its breeding grounds in the Arctic all the way to its sub-Antarctic wintering grounds.

The majority of migratory species, namely songbirds and shorebirds, migrate at night, alone, and without the guidance by parents or other experienced individuals.

Franz Bairlein

franz.bairlein@ifv-vogelwarte.de

Wolfgang Wiltschko

wiltschko@bio.uni-frankfurt.de

1 Institute of Avian Research, An der Vogelwarte 21, 26386 Wilhelmshaven, Germany

2 Fachbereich 15 Biowissenschaften, Goethe Universität Frankfurt am Main, Campus Riedberg, Max-von-Laue-Str. 13, 60438 Frankfurt am Main, Germany
Nevertheless, they find their way and do arrive at their species-specific wintering range. Thus, their migratory abilities must be innate. This has been shown in a number of studies with different bird species. Obviously, these migrants are equipped with the innate knowledge of both the proper timing of their migration and the compass direction in which they have to go. They are also able to use compass mechanisms for finding their ways and, not the least, to cope with the remarkable physiological requirements allowing them to fly non-stop for many hours. Similarly fascinating are the capabilities of the very long-distance migrants to navigate home or to a previous wintering site with the extraordinary precision of a few meters after having covered a distance of $10,000 \mathrm{~km}$ or more.

Initially indicated by ringing birds with individually numbered bands and by their recoveries, recent developments in technology now allow documenting the actual flight tracks of birds, revealing the vast amount of their movements in considerable detail. Migratory routes and individual flights can now be remotely recorded by new technologies such as satellite tracking, GPS-tracking or light-level geolocation, the latter even being applicable to rather small birds. In larger bird species, these devices can also record inflight behaviour and physiology, thus making entirely novel insights in migration biology possible. In addition, recent developments of new techniques in other fields such as neurobiology, molecular biology, biochemistry and genomics together with the availability of data banks open up new opportunities to use different data from various sources in combination, to analyse new points of view and thereby to promote the rapid deepening of our understanding of the various aspects of bird migration.

Yet, despite the increasing knowledge gathered over the last few decades, we still lack a coherent understanding of bird migration. The present Special Issue is to provide 
insights into some of the recent discoveries. It does not aim to provide an extensive review of the existing knowledge on bird migration, as that is summarized in quite a few recent books. Rather, our Special Issue is presenting in a kaleidoscopic manner mini-reviews on some selected topics of current interest and perspectives for future research in the study of various fascinating phenomena of bird migration. A particular emphasis is on physiological and navigational aspects of migrating species. The 14 contributions are divided into two groups, with one focusing on the internal control of the migration status and the other on the flight itself. The five papers in the first part present information on the genetics of migration, on hormones, energy metabolism, neuronal control of timing and the influence of energy stores on the bird's decision whether to leave or to stay. The nine papers in the second part focus on the migration flight itself, highlighting the influence of atmospheric conditions, the various aspects of compass mechanisms and navigation and their neuroanatomical basis. They also deal with route simulations and the novel techniques of tracking and biologging, presenting first results.

We, the Guest Editors, are very thankful to the Editorin-Chief for his idea and support of that Special Issue on "Bird Migration" of the Journal of Comparative Physiol$\operatorname{ogy} A$. We are also very grateful to all the authors, who happily agreed to contribute to the Special Issue and provided interesting, stimulating papers, irrespective of the many time-constraints we all face. Thanks are also due to the reviewers for their constructive comments and their many helpful suggestions from which this issue profited. 